\title{
Production of stable membrane proteins for the development of Biologics
}

\author{
Stephanie Duclos ${ }^{1}$, Nicolas Bertheleme ${ }^{1}$, Rahul Yadav ${ }^{1}$, Richard Hitchman ${ }^{1}$, \\ Shweta Singh ${ }^{1}$, John Barker ${ }^{1}$, Dirk Ullmann ${ }^{2}$ \\ ${ }^{1}$ Evotec (UK) Ltd, ${ }^{2}$ Evotec AG
}

The delivery of membrane proteins in a sufficient amount and suitable quality is considered to be the bottleneck of membrane protein-based drug discovery. To overcome the challenge of expressing and purifying stable and functional membrane proteins such as transporters, receptor or channels, Evotec offers a robust membrane protein platform, complemented with expertise in biophysics (e.g. X-Ray crystallography) and bioassays (Ligand-binding assay). Our comprehensive workflow includes construct design, recombinant expression in different expression systems, and a modular approach to construct triage and detergent screening. This pipeline has recently been utilized for the identification of a stable G Protein-coupled receptor (GPCR) that maintained the integrity of its epitope and could be recognised by antibody binding in a cell-based assay. 